\title{
LA REPRODUCTION INTERDITE : ENJEUX NARRATIFS DU TRANSFERT IDENTITAIRE CHEZ MAGRITTE ET ANTONIONI
}

\author{
Dominique Nasta ${ }^{1}$
}

L'amateur d'art ou le spectateur averti auront sans doute marqué leur étonnement à la lecture du titre de notre contribution.

Pourquoi tenter un rapprochement entre Magritte et Antonioni ?

Magritte n'appréciait guère le cinéma moderne et souvent élitiste (les films de Laurel et Hardy et Babette s'en va-t-en guerre figuraient parmi ses titres préférés), alors qu'Antonioni cite volontiers les noms de Braque ou de Matisse, mais ne semble pas avoir des affinités particulières avec le surréaliste belge...

La clé de l'exercice comparatif auxquel nous allons nous livrer dans les pages qui suivent, se trouve dans ce que l'exégète allemand Ralf Konerssmann a appelé "la visibilité de la pensée"2. Aussi bien Magritte qu'Antonioni ont en commun cette volonté persistante de donner vie à la pensée, à l'univers mental, à travers des images, statiques ou en mouvement. Mieux encore, les deux créateurs visent la mise en place d'un véritable univers narratif élaboré avec ces mêmes

1 Professeur à l'Université Libre de Bruxelles.

2 Cf. R. Konerssmann, René Magritte, Die Verbotene Reproduktion Über die Sichtbarcheit des Denkens, Fisher Verlag, 1991, p. 6-7. 
images. Un univers peuplé de doubles, de miroirs sans reflet, d'observateurs observés, d'objets dé-familiarisés, de lieux qui gardent la trace des humains longtemps après leur disparition, en défiant l'espacetemps.

En parcourant l'essai de Konerssmann sur la métaphore conceptuelle chez René Magritte : Sur la visibilité de la pensée, on est frappé par la grande ressemblance entre la philosophie magrittienne du double sens et le mécanisme de fonctionnement de la diégèse dans l'œuvre de Michelangelo Antonioni.

La philosophie du double sens met en scène la normalité démonstrative de l'image et un langage au sens souvent mystérieux, voire insidieux. L'on arrive ainsi à une démystification des prototypes (idées reçues, clichés) qui perturbe un monde aux lois pré-établies.

C'est dans la disposition du visuel et du verbal que s'articule le savoir narratif, car le spectateur saisit le sens uniquement après avoir compris le pourquoi d'une énumération de prototypes. Il importe que le processus d'observation visuelle soit actif et non pas passif et que les connexions qui amorcent le sens ultime se fassent non seulement par le montage, mais aussi à l'intérieur des images elles-mêmes, à l'aide de différents embrayeurs.

Le caractère actif de la démarche d'observation ne se traduit pas uniquement par la "saisie" d'un sens caché, occulté, faussement simplifié. Ce processus actif implique la prise en compte d'une expérience que l'on a acquise dans le passé en contemplant le monde réel ${ }^{1}$.

Lorsqu'il y a conflit entre le texte ("Ceci n'est pas une Pomme") et l'image ("voici une pomme") chez Magritte, un troisième sens doit naître, après réflexion. Il s'ensuit que la réflexion de celui qui contemple devrait donner lieu à plusieurs variantes : ceci est un mensonge, une invention, la destruction d'une idée reçue, etc.

Entre les mots et la pensée qui conceptualise ces mots, il y a l'intention d'établir un sens.

Dans Meaning in Film, nous nous attachions à démontrer que la signification non-naturelle est celle qui exprime le mieux, au niveau visuel, le mélange entre une intention de transformer le sens d'un objet ou d'une personne et la reconnaissance de cette intention par le spectateur-récepteur. Une action à signification non-naturelle repose

1 Cette problématique a déjà fait l'objet d'une communication que j'ai tenue à l'occasion du "Colloque Antonioni" organisé par le Festival du Film de Genève en octobre 1993 et dont le titre était Antonioni et la métaphore conceptuelle. 
sur la capacité du public de percevoir mais aussi et surtout de reconnaître une intention au sein de la vision ${ }^{1}$.

Comment rendre compte de l'utilisation d'une figure de la pensée au sein d'une narration, fût-elle filmique ou picturale ?

On retrouve, dans les écrits de Magritte comme dans ceux, plus ponctuels, d'Antonioni, un même souci de voir autrement la réalité, et donc d'évoquer le mystère, les affinités secrètes qui existent entre des images au demeurant très, voire trop communes.

Ce qu'ils semblent vouloir transmettre au spectateur-observateur équivaut à un grand point d'interrogation quant au bien-fondé de la représentation, de la reproduction imagée de la réalité.

Pour Magritte, comme pour Antonioni, il n'y a pas de reproduction artistique du fait réel, soit-il personnel ou collectif, sans l'intervention de la pensée imagée. Une représentation artistique qui n'engage pas une tension cognitive, une méditation sur l'être et sur sa connaissance manifeste du monde, s'avère plate, sans consistance, sans véritable mystère à décoder.

Selon Magritte, "penser à une image signifie voir une image" et "la peinture (...) n'est pas orientée par des recherches formelles mais concerne la pensée, exclusivement par son manque de solution avec le monde et son mystère"2. Dans une interview télévisée de 1966, il déclarait :

Toute chose visible cache autre chose de visible (à propos de La réponse inattendue) ; (...) le mystère est quelque chose de méconnaissable qui provoque de la joie, car nous ne sommes pas dépaysés, nous sommes réveillés ${ }^{3}$.

$\mathrm{Vu}$ que l'image magrittienne fonde sa lisibilité imagée sur une dialectique de type surréaliste faisant alterner le visible et l'invisible, toute opération de substitution devient possible. A l'occasion d'une conférence faite à Londres à la fin des années vingt, Magritte posait déjà les jalons de sa philosophie visuelle :

1 Cf. D. NASTA, Meaning in Film : Relevant Structures in Soundtrack and Narrative, Berne, Peter Lang, 1991, p. 19-21.

2 In R. MagritTe, Écrits complets, édité par A. Blavier, Paris, Flammarion, 1992, p. 108.

3 Cf. Document de l'I.N.A. extrait de l'émission Terre des Arts, 1966, Int. de MaxPol Fouchet. 
Un mot peut remplacer une image. Une image peut remplacer un mot. Un objet réel peut remplacer un mot... Il existe une affinité secrète entre certaines images. Elle vaut également pour les objets représentés par ces images... Il est possible d'obtenir une image nouvelle qui résistera mieux à l'examen du spectateur ${ }^{1}$.

L'intention constante de rendre visible la pensée même de l'existence des êtres et des choses est redevable à l'Italien De Chirico, premier peintre à avoir osé mettre en doute l'identité familière du monde. Les créatures sans visage, dépourvues de vue, aux corps tronqués, errant dans des paysages désertifiés, évoquent une angoisse métaphysique que 1'on retrouve chez Magritte, mais aussi dans les ensembles d'images définitoires de l'univers antonionien.

Toutefois, contrairement à De Chirico, Magritte se défie des symbolisations psychanalytiques et préfère arborer une normalité de type hyper-réaliste de la représentation. L'énigme, le mystère, naissent du décalage entre une description fidèle de l'univers représenté et un titre métaphorique, voire allégorique, associé (on pourrait presque dire "apposé") à cet univers. C'est la relation incongrue entre une image et un mot qui incite l'observateur à une interprétation active.

A l'instar de Magritte, Breton n'admet pas la persistance d'un regard passif : "J'affirme que l'œil n'est pas ouvert aussi longtemps qu'il se limite au rôle passif d'un miroir".?

Antonioni a toujours saisi, lors de ses témoignages sous forme d'interviews ou à travers ses propres écrits, l'enjeu considérable de la mise en images d'idées qui ne doivent pas nécessairement être doublées de paroles ou de symboles visuels ouverts, facilement identifiables. Ce qui ressort avec évidence de ses propos, c'est précisément cette volonté de faire participer le public activement à l'élaboration d'un sens qui ne se donne pas à voir tout de suite, mais qui est bien là, prêt à être découvert.

Dans un article publié en 1942, dans la revue italienne "Cinema", intitulé Suggestions de Hegel, il écrit :

Le cinéma est un art fondamentalement figuratif, et comme la peinture, son moyen de représentation formelle est l'apparence

1 Cf. Écrits complets, op. cit., p. 360.

2 In I. HEDGES, Languages of revolt : Dada and surrealist literature and film, Durham N.C., Duke University Press, 1983, p. 119. 
extérieure de la nature et des individus, à condition que celleci laisse deviner leur intérieurité .

Vingt ans plus tard, lorsque sa trilogie filmique ( $L$ 'Avventura, $L a$ Notte, L'Eclisse) ébranle la critique et le public, Antonioni poursuit sa réflexion, mettant l'accent sur la tension filmique créée entre les éléments spatio-temporels et les images qui les engendrent :

Voir pour nous, c'est une nécessité. Pour un peintre aussi, le problème est de voir. Ce qui a un sens aujourd'hui pour nous ce sont les rapports spatiaux et temporels qu'entretiennent ces éléments et la tension qui se forme entre eux ${ }^{2}$.

Enfin, lors de la sortie de Professione Reporter, le cinéaste insiste sur la préoccupation qui a toujours été au centre de sa création, à savoir l'élaboration d'un sens nouveau, capable d'interpeller différemment le spectateur :

Il est clair que je dois regarder le monde d'un oil différent, je dois chercher à le pénétrer par des sentiers non-battus, donc tout changer pour moi, la matière narrative, les histoires, la finalité des histoires, et il ne peut en être autrement si je veux anticiper, tenter d'exprimer ce que je crois arriver $(. . .)^{3}$.

La thématique du transfert identitaire, qui opère en peinture comme au cinéma, par dédoublements et substitutions, est au cœur de la démarche créatrice du peintre et du cinéaste. Il ne s'agit toutefois pas uniquement de prises de rôles limitées à la sphère humaine : la nature, les objets, les ensembles architecturaux, peuvent également faire l'objet d'un déplacement, d'une transformation.

Pour Magritte et Antonioni le monde vu est multiple, pluriel, recomposable à l'infini. L'espace-temps démultiplié par les effets picturaux du surréaliste belge ou par les mouvements imprévisibles de la caméra "voyageuse" du cinéaste italien (ce que les Anglo-saxons appellent "the wandering camera"), se double d'une dimension allégorique, dont la portée métaphysique demeure parfois insaisissable.

1 Repris dans M. AnTonioni, Écrits (vol. 4), 1936-1985, édité par G. TinAzZI, Rome, Cinecittà Int., 1991, p. 171-177.

2 Cf. interview dans La Stampa, juin 1963. Repris dans M. ANTONIONI, Entretiens et Inédits (vol. 5) 1950-1985, édité par G. TINAZZI et C. Dr CARLo, Cinecittà, Rome, 1992.

3 Ibid. Inverview dans L'Europeo, déc. 1975. 
Plusieurs toiles de Magritte, exécutées vers la fin des années vingt, auraient pu servir d'embrayeur narratif au type de récit proposé par Antonioni dans L'Avventura (1960).

Il s'agit notamment de La chambre du devin, qui traite de la thématique de la disparition et des conséquences psychologiques d'un tel phénomène : l'on y voit une silhouette en carton à l'avant-plan d'une toile brisée dont le centre laisse apparaitre une ombre semblable à la silhouette.

Si nous nous arrêtons un instant sur la séquence d'ouverture de $L$ 'Avventura, nous nous apercevons de la dimension non-naturelle des scènes qui dégagent un sens double, aussi bien dans la visualisation du transfert identitaire que dans les dialogues. Lorsque Anna (Lea Massari) fait l'amour avec Sandro et qu'elle dit "qu'elle va mal", la construction en profondeur de champ et le surcadrage nous font découvrir Claudia (Monica Vitti) à l'arrière-plan, en attente dans la cour. Il y a donc à l'intérieur de l'espace réel un espace construit qui produit un sens second, confirmé par après : Claudia remplacera Anna, qui n'est plus satisfaite de son existence.

Dans Les traces vivantes, les composantes d'un paysage (arbre, pierres, nuages) sont encerclées et définies par des mots correspondant à d'autres notions : sur l'arbre on peut lire notamment "femme nue". Il est difficile de ne pas songer, lors de la contemplation de ce tableau de Magritte, à la scène de recherche sur l'île, après la disparition soudaine d'Anna, lorsque tout indice naturel ou objectuel marque la trace du personnage absent.

De la même manière, La malédiction (1931) nous fait découvrir un ciel envahi par des nuages menaçants, dépourvu de toute présence humaine ou objectuelle. C'est le vide après la disparition, la porte ouverte aux questionnements de toutes sortes : dans L'Avventura, un ciel de plomb, délimité par le cadre filmique de manière analogue, surplombe l'île et ses personnages à la dérive.

Avant la disparition d'Anna,le transfert d'identité est illustré par une métaphore courante chez Antonioni : la prise de vue de dos qui entraîne un rapport de similitude issu d'une grande complicité entre personnages. Le dialogue porte sur une blouse : "Laquelle tu mets ?" demande Claudia.

Dans L'image parfaite, Magritte opère une variation sur ce que sera plus tard La reproduction interdite : une femme au buste nu regarde de dos un cadre vide. 
L'anticipation du transfert identitaire s'opère aussi par l'élément de la blouse échangée. En voyant Vitti porter la blouse de sa fille disparue, le père d'Anna l'interpelle. Elle répond : 'Oui, c'est à Anna. Elle me l'avait donnée hier. Je ne voulais pas... mais je n'en avais pas d'autre". Le spectateur actif comprend, reconnaît l'intention de l'auteur bien avant que soit détaillée l'histoire d'amour avec Sandro et la nécessité de la disparition annoncée d'Anna.

Antonioni fait appel aux miroirs pour représenter le thème du double sexuel : Claudia qui a déjà été identifiée à Anna par le don de la blouse, essaye une perruque noire sous le regard indulgent de Patrizia, qui elle-même prend une perruque blonde. Claudia observe Patrizia de dos à travers le miroir, mais il y a un deuxième miroir à deux panneaux. Patrizia pousse l'un des panneaux et semble effacer Claudia. Mais Claudia ré-apparaît et Patrizia lui dit: "Tu as l'air de quelqu'un d'autre".

Une toile plus tardive de Magritte, Les liaisons dangereuses (1936), nous montre une femme nue de profil à la chevelure abondante, tenant entre ses mains un miroir qui occulte son corps jusqu'aux hanches; le miroir dévoile la partie du corps cachée par le cadre, mais en position inverse.

Le choix de L'Eclisse (1962) trouve sa justification dans le potentiel considérable d'espaces de substitution que le film met en scène, tout en conservant une simplicité extrême dans l'évocation des faits et des états psychologiques des personnages.

Après une séparation qui marque la tonalité du récit, Vittoria (Monica Vitti) est confrontée au monde chaotique de la Bourse : la rencontre avec un nouvel amour va se faire à l'intérieur d'un même espace aux colonnes massives, qui écrasent l'individu.

Ce qui frappe en outre dans cette séquence, c'est son caractère de matrice narrative, de synthèse annonciatrice de la suite des événements. Le spectateur averti saisit simultanément l'attrait réciproque des deux protagonistes et leur véritable différence, que la séparation finale ne peut que confirmer. D'autre part, Antonioni suggère par l'évocation hyper-réaliste du monde de la Bourse, la nécessité de l'existence d'un autre univers. Ce dernier sera substitué par plusieurs espaces extérieurs qui ne subiront aucune contrainte "matérielle" et qui se videront peu à peu de la dynamique chaotique du mouvement humain. 
Le dépassement du visuel et du verbal par une métaphore qui déclenche un raisonnement va se concrétiser dans L'Eclisse par les espaces de substitution. La longue séquence finale repose sur deux aspects essentiels.

Le premier relève de que Seymour Chatman définit comme étant la stase, processus qui implique l'arrêt de tout phénomène et qui se double ici de la relation "arrêtée" des personnages, malgré leur promesse de se revoir -ce serait l'éclipse de leur relation'.

Antonioni reprend des endroits montrés précédemment dans le film lors des rencontres entre les deux protagonistes. Le seau plein d'eau se vide, le robinet s'arrête, les feuilles d'arbres ne bougent plus et la géométrie des bâtiments devient oppressive. Le spectateur croit que quelque chose de grave est arrivé ; ses craintes sont confirmées par la toute dernière séquence où les réverbères du quartier s'allument et une lampe aveuglante rappelle l'éclipse du soleil sur fond de bruit musicalisé.

Peu avant, le cinéaste nous donne à lire de façon quasiostentatoire le journal qu'un homme est en train de parcourir. Il y a deux titres : "Compétition atomique" et "La Paix est fragile".

Le deuxième volet du final de L'Eclisse est peut-être celui qui rend le fragment beaucoup moins abstrait. Ce volet marque à quel point l'hypothèse atomique n'est qu'une possibilité, une hypothèse narrative parmi d'autres. Il insiste toutefois sur un phénomène beaucoup plus difficile à saisir métaphoriquement : la disparition, non pas de l'ensemble de l'humanité mais du protagoniste de l'histoire. L'on voit de dos une fille blonde descendre du bus ; la caméra la suit, pour ensuite découvrir que ce n'est pas Vittoria. Les panoramiques dans la rue révélant des personnages sans importance suggèrent un rapport symbolique au sein duquel tout individu peut être associé à une autre identité.

Vittoria et Piero vont céder la place à un autre personnage, à une autre histoire, et ainsi de suite, ad infinitum ${ }^{2}$.

Les similitudes entre l'univers à forte connotation métaphysique de L'Eclisse et plusieurs toiles de Magritte abordant la même problématique sont troublantes. Comme avant lui De Chirico, Magritte utilise la perspective de l'infini, le point de fuite et une forte

1 In S. ChATMAN, Antonioni or the Surface of the World, Berkeley, University of California Press, 1985.

2 Voir à ce sujet mon analyse plus détaillée de L'Eclisse dans Meaning in Film, op.cit., p. 132-144. 
angularité du cadre pictural. Ce dernier est dépourvu de personnages, mais "habité" par leur présence passée. Selon Magritte, "il s'agit d'une nouvelle vision, où le spectateur retrouve son isolement et entend le silence du monde" 1 .

Ainsi, dans Le village mental (1926), un coin de rue en perspective angulaire est délimité par une porte de maison d'un côté et par une série de fenêtres de l'autre, alors qu'un rideau séparateur pendu à un rail domine l'avant-plan du tableau. L'association, fût-elle arbitraire, entre le titre et la toile, incite à l'élaboration de nombreux présupposés narratifs.

Les Amants (dont il existe plusieurs versions) semblent, d'après Magritte, "s'inspirer de plans cinématographiques" : on y voit deux têtes rapprochées, occultées par des draps blancs; les sujets scrutent l'horizon, sur fond de paysage arboré. Ou bien, dans une autre version, les visages masqués par les draps s'embrassent dans une pièce aux plafonds visibles. L'intention de signification est claire, elle repose sur une possibilité infinie d'attributions identitaires, mais aussi sur la relativité de la notion de lien amoureux dans un monde aux allures changeantes.

On songe à l'étreinte désespérée de Piero et Vittoria à la fin de L'Eclisse, peu avant leur séparation finale.

Il importe de rapprocher le final de L'Eclisse et la signification non-naturelle qui en découle, de Blow up (1967). Le point commun partagé par les deux films, malgré les moyens différents mis en œuvre, réside dans la capacité de recréation analogique suggérée par Antonioni. Si disparition il y a, si cassure, séparation, brisure, échec s'ensuivent, un nouveau sens apparaittra selon de nouvelles conventions, codifiées de façon réaliste ou allégorique.

A travers Blow up, Antonioni détaille le côté "révélateur" du dispositif photographique et suggère, par l'évidence de ce qui se donne à voir, le caractère relatif de toute recherche de vérité. A partir d'une réalité donnée, les pistes narratives se multiplient sans fournir une réponse définitive.

Il est important de constater à quel point les réflexions de Magritte sur la reproduction artistique d'un monde donné se rapprochent de la pensée visuelle d'Antonioni, telle qu'elle se donne à

1 Cf. D. Sylvester (éd.), René Magritte, Catalogue raisonné, vol. I, Paris/Anvers, Flammarion/Fonds Mercator, 1992. 
voir dans Blow up. Le peintre nous livre, dans ses Écrits, la clé de l'élaboration de l'univers qui lui est propre :

Je finis par trouver dans l'apparence du monde réel lui-même la même abstraction que dans les tableaux... je devins peu certain de la profondeur des campagnes, très peu persuadé de l'éloignement du bleu léger de l'horizon, l'expérience immédiate le situant simplement à la hauteur de mes yeux. Il me fallait maintenant animer ce monde qui, même en mouvement, n'avait aucune profondeur et avait perdu toute consistancel.

Dans Blow up le rapport entre deux personnages est remplacé, au niveau narratif, par le rapport entre un personnage et la réalité fragmentée par la pellicule photographique. La nature du transfert est plus globale que dans les films précédents, car il s'agit, pour Thomas, d'un passage volontaire de la réalité "sans profondeur" au monde imaginaire des mimes simulateurs.

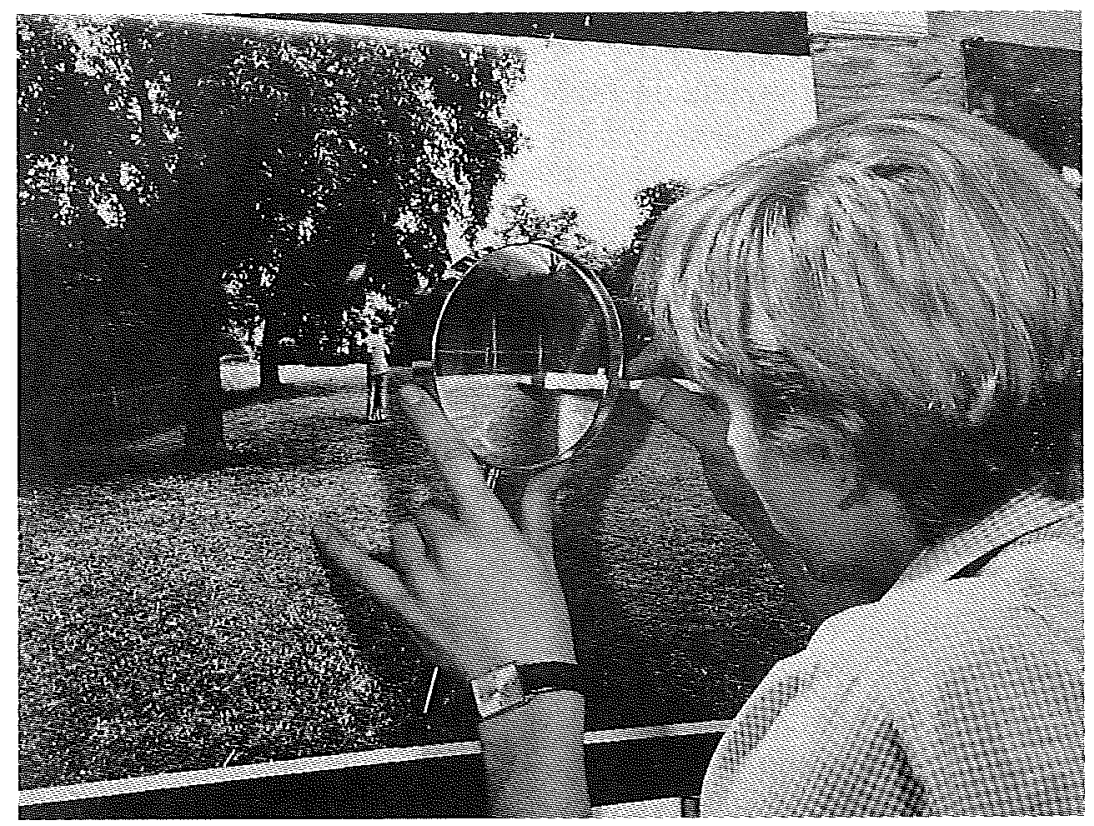

Michelangelo Antonioni, Blow up

${ }^{1}$ In Écrits complets, op. cit., p. 15. 
L'absence d'intrigue conventionnelle sert à renforcer la vision morcelée qu'a Thomas de la réalité. Un montage de type disjonctif permet à Antonioni de développer un récit (la découverte d'un fait mystérieux par un photographe et la recherche de la solution à l'énigme), tout en ouvrant des parenthèses de pure observation sociétale, sans incidence sur la suite des événements : le protagoniste rencontre des vieux hères dans une usine désaffectée, prend beaucoup de clichés de mannequins luxueux, achète une hélice géante chez un antiquaire, etc. Ces parenthèses permettent au spectateur de se remémorer l'expérience rétroactive de Thomas lorsqu'il est confronté au décodage photographique d'un monde qu'il ne mâtrise pas.

Pendant que Thomas analyse la chronologie de l'événement qu'il pense pouvoir déceler, le spectateur a droit à une double perception : celle du personnage observateur et celle, plus globale, du cinéaste. Le résultat de l'enquête photographique débouche, comme dans tout faux policier conçu par Antonioni, sur un non-lieu. Le cadavre identifié sur la photo est repéré dans le parc, pour ensuite disparaître sans laisser de traces. A partir de cet instant il y a transfert allégorique de la sphère du visible, dont Thomas s'avère insatisfait, à celle de l'invisible, reproductible à l'infini.

Grâce à la force du geste mimique, une véritable partie de tennis s'engage entre une bande masquée de mimes et Thomas; les spectateurs entendent d'ailleurs les coups rythmés de la balle invisible. A l'improviste, Thomas disparaît et on ne voit plus que la pelouse verte ; Thomas devient le quatrième élément (cadavre, balle, appareil photo) des objets qui apparaissent et disparaissent.

Pour l'exégète George Slover, l'épiphanie de la partie de tennis est l'initiation de Thomas au mystère de son art. Le cadavre que seul son appareil a créé n'est réel que sur pellicule : par le geste de la disparition sur la pelouse, Antonioni nie l'existence de Thomas en dehors du circuit de l'art, qui modèle la réalité à partir d'une simulation considérée comme réelle ${ }^{1}$.

1 Cf. G. Slover, "Blow up : média, message, mythe et fiction", The Massachussetts Review, n 9, automne 1968, p. 753-770. 


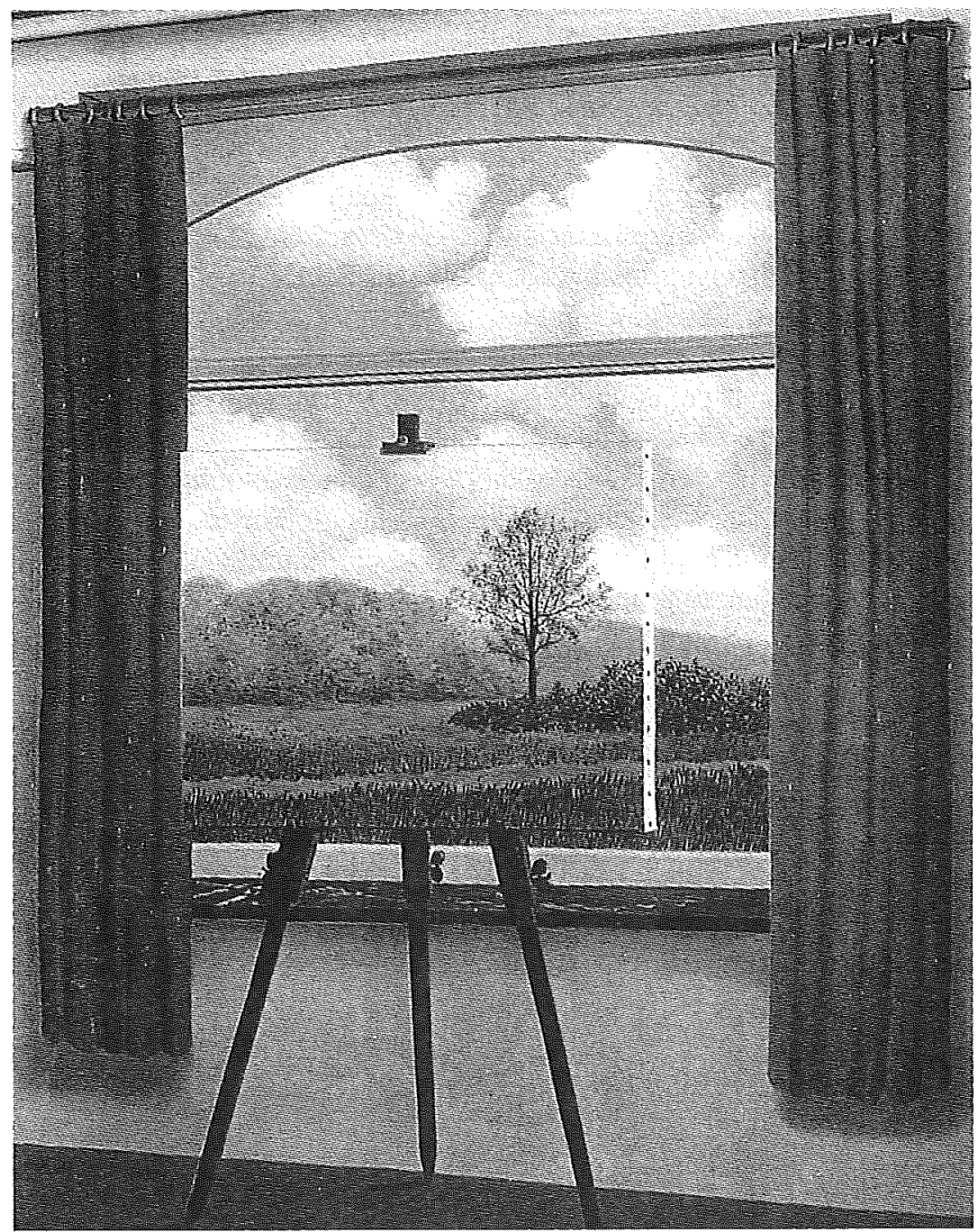

René Magritte, La condition humaine (C) Charly Herscovici c/o SABAM Belgium 
Lorsqu'il peint, en 1926, Le seuil de la forêt, où le milieu d'un tronc d'arbre laisse apparaitre un fragment de mur en brique, Magritte s'explique :

Appeler «arbre» l'image d'un arbre est une erreur, une confusion sur la personne, puisque l'image d'un arbre n'est pas assurément un arbre. L'image est séparée de ce qu'elle montre ${ }^{1}$.

Le paysage isolé (1928) donne à voir un personnage de dos, cadré jusqu'aux épaules, regardant au loin une maison sur une colline entourée d'arbres. De sa bouche sort une vignette, qui équivaut à un constat de refus de la convention picturale : "je ne vois rien autour du paysage".

Toutefois, c'est dans la conception de la célèbre Condition humaine (1933) et de ses nombreuses variantes, que Magritte préfigure de la manière la plus évidente la problématique visuelle du double sens orchestrée par Antonioni dans Blow up.

Le peintre semble fasciné par l'existence d'un même objet dans deux espaces différents, à la fois dans le passé et dans le présent d'un moment identique. Au sein d'une mise en scène typique du tableau dans le tableau, la toile située devant la fenêtre d'une chambre délimite le paysage extérieur par son propre cadre. "L'essentiel -affirme Magritte dans une lettre à Breton de 1934- était de supprimer la différence entre la vue qu'on a de l'intérieur et de l'extérieur d'une chambre."

Ce que la mise en scène de La condition humaine interroge, c'est précisément le processus de reproduction figurée du réel. Comme dans Blow up, l'image construite relativise la cö̈ncidence entre représentant et représenté.

La situation narrative de Professione Reporter (1975) repose entièrement sur les conséquences du transfert identaire et offre un exemple de forte lisibilité de la pensée antonionienne. Tout en étant beaucoup plus explicite que dans les réalisations antérieures, la dynamique visuelle de substitution proposée par le cinéaste défie plus que jamais toute norme représentationnelle.

Locke, le héros principal devenu effectivement un autre, imagine pouvoir fonder sur cette substitution une nouvelle vie. Mais

1 Extrait d'une lettre de 1959 à A. Bosmans. 
l'existence de l'autre ne lui appartient pas et il fait semblant de jouer un rôle qui n'est pas le sien. Par conséquent, son drame se déroule dans la perte de points de repère qui pourraient donner un sens à ses actions.

$\mathrm{Vu}$ que Locke ne souhaite pas que sa véritable identité soit révélée, son regard et ses pensées subjectives s'inscrivent dans une dichotomie spatiale qui empêche tout conditionnement temporel. Le flash-back devenu "glide-back" (glissement en arrière, selon la terminologie proposée par Seymour Chatman) permet à Antonioni de montrer les deux hommes ensemble, et donc leur ressemblance frappante de carrure et de traits. Ce procédé souligne la fragilité et la relativité de la vie.

Antonioni privilégie une nouvelle fois le mystère d'une situation "doppelgänglich".

Le mystère de ce double est omniprésent dans la production artistique de Magritte. Sa mise en image et "en texte" constitue une exception, dans la mesure où, assez souvent, le titre du tableau correspond à ce qui est montré. C'est le cas, notamment, de $L a$ Décalcomanie et de La Reproduction interdite. Dans le premier, le même personnage est montré dans la différence de son opacité et de sa transparence. Le deuxième représente Edward Jones, amicollectionneur du peintre, placé devant un manteau de cheminée surmonté d'un miroir : dans ce miroir apparaît la visibilité noninversée du reflet du personnage. Ce face à face qu'autorise le miroir s'avère être un interdit, un territoire non-transgressable ${ }^{1}$.

C'est ce même interdit qui frappe, à un autre échelle, le héros antonionien qui s'obstine à dire "My name is Robertson". Sa nouvelle identité dépend d'accessoires fragiles (moustache, agenda, lunettes) et ne cesse pas d'être remise en question par ceux et celles qui l'ont connu.

A la jeune fille qu'il rencontre sur son trajet odysséen, il explique : "J'étais quelqu'un d'autre, mais je l'ai échangé". Plus tard, son parcours achevé dans la désillusion, c'est toujours à elle qu'il raconte la parabole de l'aveugle qui, deçu par le monde qu'il voit après avoir été guéri, se suicide.

L'homme démultiplié, copié, dé-calqué, se retrouve également dans la longue série magrittienne du monsieur au chapeau-melon;

1 Voir à ce sujet l'étude de R. JongEN, René Magritte ou la pensée imagée de l'invisible, Bruxelles, Facultés Universitaires Saint-Louis, 1994. 
cette thématique apparaît souvent en rapport avec la notion de paysage et de voyage, comme nous l'indiquent les titres: Les grands voyages, Le chemin de Damas ou encore Le chef-d'ouvre ou les mystères de l'horizon. Ce qui semble intéresser aussi bien le peintre que le cinéaste, c'est comment placer des personnages dans un paysage en établissant plusieurs types de significations, plusieurs catégories de questionnements.

C'est dans ce caractère illimité, réversible, polyphonique du sens, que se trouve l'essence de la comparaison entre la narrativité moderne antonionienne et le mystère imagé des tableaux de Magritte. 